\title{
APPROXIMATION OF ENTIRE FUNCTIONS OF SLOW GROWTH
}

\author{
Ning Juhong ${ }^{1}$, Chen Qing ${ }^{2}$ \\ ${ }^{1,2}$ College of Mathematics and Information Science \\ Jiangxi Normal University \\ Nanchang, Jiangxi, P.R. CHINA
}

\begin{abstract}
In this paper, first of all, we defined the generalized order and generalized type of Taylor entire function; Secondly, we show some interesting relationship on the maximum modulus, the maximum term and the coefficients of Taylor entire function; Finally, we study the polynomial approximation of entire functions in Banach spaces $((B(p, q, k) ; f)$, Hardy spaces, Bergman spaces), the coefficient characterization of generalized type of Taylor entire function of slow growth has been obtained in terms of the approximation errors.
\end{abstract}

AMS Subject Classification: 30B50

Key Words: Taylor entire function, generalized order, generalized type, approximation error.

\section{Introduction and Notation}

The growth and the value distribution of Taylor entire function were studied for a long time. Many researches have done in-depth research and many important results, which have been obtained in ${ }^{[1-8]}$. For example, S. M. Shah ${ }^{[1]}$ and S. K. Bajpai ${ }^{[2]}$ gave some different characterizations on the coefficients and the maximum modulus, the maximum term, and the rank of the maximum term for the Taylor entire function of fast growth $\rho=\infty$. On the other hand, G. P. Kapoor, A. Nautiyal ${ }^{[3-4]}$ and R. Ganti, G. S. Srivastava ${ }^{[5]}$ continued this

Received: $\quad$ September 25, 2016

Revised: December 25, 2016

Published: $\quad$ March 28, 2017

$\S_{\text {Correspondence author }}$ (c) 2017 Academic Publications, Ltd. url: www.acadpubl.eu 
work and defined generalized order and generalized type for the Taylor entire function of slow growth $\rho=0$ in a new way, as follows:

Let $\bar{\Omega}$ denote the class of functions $h(x)$ satisfying the following conditions $(\mathrm{H}, \mathrm{i})$ and $(\mathrm{H}, \mathrm{ii})$.

$(\mathrm{H}, \mathrm{i}) h(x)$ is defined on $[a, \infty)$ and is positive, strictly increasing, differentiable and tend to $\infty$ as $x \rightarrow \infty$;

$(\mathrm{H}, \mathrm{ii})$

$$
\lim _{x \rightarrow \infty} \frac{d(h(x))}{d(\ln x)}=k(0<k<\infty) .
$$

Then R. Ganti and G. S. Srivastava ${ }^{[5]}$ defined generalized order $\rho(\alpha, f)$ and generalized type $\tau(\alpha, f)$ of an entire function $f(z)$ as:

$$
\rho(\alpha, f)=\limsup _{r \rightarrow \infty} \frac{\alpha(\ln M(r))}{\alpha(\ln r)}, \tau(\alpha, f)=\limsup _{r \rightarrow \infty} \frac{\alpha(\ln M(r))}{[\alpha(\ln r)]^{\rho}},
$$

where $\alpha(x) \in \bar{\Omega}$.

In this paper, we defined generalized order and generalized type is essentially due to R. Ganti and G. S. Srivastava ${ }^{[5]}$, but we extend the range of $\alpha(x)$, and then extend some know results of entire function of slow growth.

Let

$$
f(z)=\sum_{n=0}^{\infty} a_{n} z^{n}
$$

be an entire function. Set $M(r) \equiv M(r, f)=\max _{|z|=r}|f(z)|, u(r) \equiv u(r, f)=$ $\max \left\{\left|a_{n}\right| r^{n}: n=1,2, \cdots\right\}$ and $v(r) \equiv v(r, f)=\max \left\{n: u(r)=\left|a_{n}\right| r^{n}\right\}$. Then $M(r), u(r)$ and $v(r)$ are called respectively the maximum modulus, the maximum term, and the rank of the maximum term of $f(z)$ for $|z|=r$. Elements in the range set of $v(r)$ are called principle indices.

Normally, the growth of $f(z)$ is measured in terms of order $\rho$ and type $\tau$ defined as under:

Definition 1. ${ }^{[5]}$ The order and the type of entire function can be defined as:

$$
\begin{gathered}
\limsup _{r \rightarrow \infty} \frac{\ln \ln M(r)}{\ln r}=\rho, \\
\limsup _{r \rightarrow \infty} \frac{\ln M(r)}{r^{\rho}}=\tau,
\end{gathered}
$$

for $0<\rho<\infty$ various researches have give different characterizations for entire function of fast growth. In this paper we defined the generalized order and generalized type in a new way with the help of general function as following: 
Let $\Lambda$ denote the class of functions $h(x)$ satisfying the conditions $(\mathrm{H}, \mathrm{i})$ and (H, iii).

(H, iii)

$$
\lim _{x \rightarrow \infty} \frac{d(h(x))}{d\left(\ln ^{[p]} x\right)}=k \in(0, \infty), \quad p \geq 1, p \in N^{+},
$$

where $\ln ^{[0]} x=x, \ln ^{[1]} x=\ln x, \ln ^{[p]} x=\ln ^{[p-1]} \ln x$, so we can easy testify that

$$
\lim _{x \rightarrow \infty} \frac{h(c x)}{h(x)}=1, \lim _{x \rightarrow \infty} \frac{h(c+x)}{h(x)}=1
$$

for every $c>0$, that is, $h(x)$ is slowly increasing.

From (H, ii) and (H, iii), we know that $\alpha(x) \in \bar{\Omega}$ is a special case of $\alpha(x) \in \Lambda$, when $p=1$.

Definition 2. Let $\alpha(x) \in \Lambda$, then the generalized order and generalized type also can be defined in this way as under:

$$
\begin{aligned}
& \rho(\alpha, f)=\limsup _{r \rightarrow \infty} \frac{\alpha(\ln M(r))}{\alpha(\ln r)}, \\
& \tau(\alpha, f)=\limsup _{r \rightarrow \infty} \frac{\alpha(\ln M(r))}{[\alpha(\ln r)]^{\rho}} .
\end{aligned}
$$

Recently, R. Ganti and G. S. Srivastava ${ }^{[5]}$ also considered the approximation of entire functions in Banach spaces. Thus, let $f(z)$ be an analytic function in the unit $\operatorname{disc} U=\{z \in C:|z|<1\}$ and we set

$$
M_{q}(r, f)=\left\{\frac{1}{2 \pi} \int_{-\pi}^{\pi}\left|f\left(r e^{i \theta}\right)\right|^{q} d \theta\right\}^{1 / q}, q>0 .
$$

let $H_{q}$ denote the Hardy space of function $f(z)$ satisfying the condition

$$
\|f\|_{H_{q}}=\lim _{r \rightarrow 1-0} M_{q}(r, f)<\infty .
$$

and let $H_{q}$ denote the Bergman pace of function $f(z)$ satisfying the condition

$$
\|f\|_{H_{q}^{\prime}}=\left\{\frac{1}{\pi} \iint_{U}|f(z)|^{q} d x d y\right\}^{1 / q}<\infty .
$$

For $q=\infty$, let $\|f\|_{H_{\infty}^{\prime}}=\|f\|_{H_{\infty}}=\sup \{|f(z)|, z \in U\}$, then $H_{q}$ and $H_{q}^{\prime}$ are Banach spaces for $q \geq 1$. Following [5], we say that a function $f(z)$ which is analytic in $U$ belongs to the spaces $B(p, q, k)$ if

$$
\|f\|_{p, q, k}=\left\{\int_{0}^{1}(1-r)^{k(1 / p-1 / q)-1} M_{q}^{k}(r, f) d r\right\}^{1 / k}<\infty,
$$


$0<p<q \leq \infty, 0<k<\infty$ and

$$
\|f\|_{p, q, \infty}=\sup \left\{(1-r)^{1 / p-1 / q} M_{q}^{k}(r, f) ; 0<r<1\right\}<\infty .
$$

It is known ${ }^{[7]}$ that $B(p, q, k)$ is Banach spaces for $p>0$ and $q, k \geq 1$, otherwise it is a Frechet space. Further ${ }^{[8]}$,

$$
H_{q} \subseteq H_{q}^{\prime}=B(q / 2, q, q), 1 \leq q<\infty .
$$

Definition 3. Let $X$ denote one of the Banach spaces defined above, the approximation errors of entire function $f(z)$ can be defined as:

$$
E_{n}(f, X)=\inf \left\{\|f-p\|_{X}: p \in P_{n}\right\},
$$

where $P_{n}$ consists of algebraic polynomial of degree at most $n$ in complex variable $z$.

For $\alpha(x) \in \Lambda, p=1$, R. Ganti and G. S. Srivastava ${ }^{[5]}$ have obtained the characterization of generalized type of $f(z)$ in terms of the error $E_{n}(f, X)$ defined above. But, For $\alpha(x) \in \Lambda, p>1, p \in N^{+}$haven't been proved so far. In the present paper, we have made an attempt to finish it.

Our main conclusions are as follows:

Theorem 1. Let $\alpha(x) \in \Lambda$, then some necessary and sufficient conditions of the entire function $f(z)$ with generalized order $\rho$ is

$$
1^{\circ} \quad \limsup _{r \rightarrow \infty} \frac{\alpha(\ln M(r))}{\alpha(\ln r)}-1=\limsup _{n \rightarrow \infty} \frac{\alpha(n)}{\alpha\left(\ln \left|a_{n}\right|^{-1 / n}\right)}, \text { for } p=1 \text {, }
$$

$2^{\circ} \limsup _{n \rightarrow \infty} \frac{\alpha(n)}{\alpha\left(\ln \left|a_{n}\right|^{-1 / n}\right)} \leq \limsup _{r \rightarrow \infty} \frac{\alpha(\ln M(r))}{\alpha(\ln r)} \leq \limsup _{n \rightarrow \infty} \frac{\alpha(n)}{\alpha\left(\ln \left|a_{n}\right|^{-1 / n}\right)}+1$,

for $p=2,3 \cdots$.

Theorem 2. Let $\alpha(x) \in \Lambda$, then the entire function $f(z)$ of generalized order $\rho, 1<\rho<\infty$, is of generalized type $\tau$ if and only if

$$
\begin{gathered}
1^{\circ} \quad \limsup _{r \rightarrow \infty} \frac{\alpha(\ln M(r))}{[\alpha(\ln r)]^{\rho}}=\limsup _{n \rightarrow \infty} \frac{\alpha(n)}{\left[\alpha\left(\ln \left|a_{n}\right|^{-1 / n}\right)\right]^{\rho-1}}, \text { for } p=1, \\
2^{\circ} \quad \limsup _{r \rightarrow \infty} \frac{\alpha(\ln M(r))}{[\alpha(\ln r)]^{\rho}}=\limsup _{n \rightarrow \infty} \frac{\alpha(n)}{\left[\alpha\left(\ln \left|a_{n}\right|^{-1 / n}\right)\right]^{\rho}}, \quad \text { for } p=2,3 \cdots .
\end{gathered}
$$


Theorem 3. Let $\alpha(x) \in \Lambda$, then some necessary and sufficient conditions of the entire function $f(z) \in B(p, q, k)$ to be of generalized type $\tau$ having finite generalized order $\rho, 1<\rho<\infty$ is

$$
\begin{gathered}
1^{\circ} \tau=\limsup _{n \rightarrow \infty} \frac{\alpha(n)}{\left[\alpha\left(\ln \left|E_{n}(B(p, q, k) ; f)\right|^{-1 / n}\right)\right]^{\rho-1}}, \quad \text { for } p=1, \\
2^{\circ} \tau=\limsup _{n \rightarrow \infty} \frac{\alpha(n)}{\left[\alpha\left(\ln \left|E_{n}(B(p, q, k) ; f)\right|^{-1 / n}\right)\right]^{\rho}}, \quad \text { for } p=2,3 \cdots .
\end{gathered}
$$

Theorem 4. Assuming that the condition of Theorem 3 are satisfied and $\xi(\alpha)$ is a positive number, some necessary and sufficient conditions for a function $f(z) \in H_{q}$ to be an entire function of generalized type $\xi(\alpha)$ have finite generalized order $\rho$ is that

$$
\begin{gathered}
1^{\circ} \limsup _{n \rightarrow \infty} \frac{\alpha(n)}{\left[\alpha\left(\ln \left|E_{n}\left(H_{q} ; f\right)\right|^{-1 / n}\right)\right]^{\rho-1}}=\xi(\alpha), \quad \text { for } p=1, \\
2^{\circ} \quad \limsup _{n \rightarrow \infty} \frac{\alpha(n)}{\left[\alpha\left(\ln \left|E_{n}\left(H_{q} ; f\right)\right|^{-1 / n}\right)\right]^{\rho}}=\xi(\alpha), \quad \text { for } p=2,3 \cdots .
\end{gathered}
$$

\section{Preliminary lemmas}

In this section, we will give some lemmas which play an important role in the proof of theorems.

Lemma 1. Let $f(z)$ is an entire function defined by (1), then

$$
\limsup _{r \rightarrow \infty} \frac{\alpha(\ln M(r))}{[\alpha(\ln r)]^{E}}=\limsup _{r \rightarrow \infty} \frac{\alpha(\ln u(r))}{[\alpha(\ln r)]^{E}}, \quad E \in(0, \infty) .
$$

Proof. Since S.M. Shah ${ }^{[1]}$, we have

$$
M(r)<3 u(r) v\left(r+\frac{r}{v(r)}\right), \quad r>r_{0}, \quad \ln u(2 e r)>v(2 r) .
$$

Therefore $\ln v(2 r)=o(1) \ln u(2 e r)$. So for sufficiently large $r>r_{0}$, we can get $\ln M(r) \leq \ln 3+\ln u(r)+\ln v(2 r) \leq(1+o(1)) \ln u(2 e r)$, hence

$$
\limsup _{r \rightarrow \infty} \frac{\alpha(\ln M(r))}{[\alpha(\ln r)]^{E}} \leq \limsup _{r \rightarrow \infty} \frac{\alpha(1+o(1) \ln u(2 e r))}{[\alpha(\ln 2 e r)]^{E}} \cdot \frac{[\alpha(\ln 2 e r)]^{E}}{[\alpha(\ln r)]^{E}}
$$

according to (4), we obtain $\limsup _{r \rightarrow \infty} \frac{\alpha(\ln M(r))}{[\alpha(\ln r)]^{E}} \leq \limsup _{r \rightarrow \infty} \frac{\alpha(\ln u(r))}{[\alpha(\ln r)]^{E}}$, On the other hand, it follows that $u(r) \leq M(r)$, this proves lemma 1 . 
Lemma 2. [9] Suppose that $\alpha(x) \in \Lambda$, and its inverse function is $\alpha^{-1}(x)$, then

$$
\limsup _{x \rightarrow \infty} \frac{\alpha\left\{x \alpha^{-1}[A \alpha(x+B)]\right\}}{\alpha(x)} \leq A+1, \quad A>0, B>0 .
$$

Lemma 3. [9] Suppose that $\alpha(x) \in \Lambda$, and its inverse function is $\alpha^{-1}(x)$, then

$$
\limsup _{x \rightarrow \infty} \frac{\alpha\left\{(x-B) \alpha^{-1}[A \alpha(x)]\right\}}{\alpha(x)} \leq A+1, \quad A>0, B>0 .
$$

Lemma 4. [9] Suppose that $\alpha(x) \in \Lambda$, and its inverse function is $\alpha^{-1}(x)$, then

$$
\limsup _{x \rightarrow \infty} \frac{\alpha\left\{x \alpha^{-1}\left\{A[\alpha(x+B)]^{\rho}\right\}\right\}}{[\alpha(x)]^{\rho}} \leq A, \quad A>0, B>0, \rho>1
$$

\section{Proof of Theorems}

The proof of theorem 1 Let $M(\sigma)=M(r), \sigma=\ln r, \lambda_{n}=n$, then the Dirichlet series can be convert to Taylor entire functions, so the proof of theorem 1 can get from theorem 1 of paper [9].

\section{The proof of theorem 2}

Case I: When $p=1, \mathrm{R}$. Ganti and G. S. Srivastava ${ }^{[5]}$ has been proved that

$$
\tau=\limsup _{r \rightarrow \infty} \frac{\alpha(\ln M(r))}{[\alpha(\ln r)]^{\rho}}=\limsup _{n \rightarrow \infty} \frac{\alpha\left(\frac{n}{\rho}\right)}{\left[\alpha\left(\frac{\rho}{\rho-1} \ln \left|a_{n}\right|^{-1 / n}\right)\right]^{\rho-1}} .
$$

using (4), we have

$$
\limsup _{n \rightarrow \infty} \frac{\alpha\left(\frac{n}{\rho}\right)}{\left[\alpha\left(\frac{\rho}{\rho-1} \ln \left|a_{n}\right|^{-1 / n}\right)\right]^{\rho-1}}=\limsup _{n \rightarrow \infty} \frac{\alpha(n)}{\left[\alpha\left(\ln \left|a_{n}\right|^{-1 / n}\right)\right]^{\rho-1}} .
$$

When $p=2,3 \cdots$, by lemma 1 , let

$$
\limsup _{r \rightarrow \infty} \frac{\alpha(\ln M(r))}{[\alpha(\ln r)]^{\rho}}=\limsup _{r \rightarrow \infty} \frac{\alpha(\ln u(r))}{[\alpha(\ln r)]^{\rho}}=\tau .
$$

we suppose $0<\tau<\infty$, then for every $\varepsilon>0, \exists r>r_{0}$, we have

$$
\alpha(\ln u(r))<(\tau+\varepsilon)[\alpha(\ln r)]^{\rho}=\widetilde{\tau}[\alpha(\ln r)]^{\rho},
$$


or $\ln u(r)<\alpha^{-1}\left(\widetilde{\tau}[\alpha(\ln r)]^{\rho}\right), \quad \ln \left|a_{n}\right|<\alpha^{-1}\left(\widetilde{\tau}[\alpha(\ln r)]^{\rho}\right)-n \ln r$. Choose $r=$ $r(n)$ to be the unique root of equation $\ln r=\alpha^{-1}\left\{\left[\frac{1}{\tau} \alpha(n)\right]^{1 / \rho}\right\}, \quad(r \rightarrow \infty \Leftrightarrow$ $n \rightarrow \infty)$, then

$$
\ln \left|a_{n}\right|^{-1 / n}>\ln r-1 \text { or } \alpha\left(\ln \left|a_{n}\right|^{-1 / n}\right)>\alpha(\ln r-1) .
$$

It follow (4) that, when $r$ is large enough, we obtain $\alpha(\ln r-1)=(1+$ $o(1)) \alpha(\ln r)$, thus

$$
\left[\alpha\left(\ln \left|a_{n}\right|^{-1 / n}\right)\right]^{\rho} \geq(1+o(1))^{\rho}[\alpha(\ln r)]^{\rho}=(1+o(1))\left[\frac{1}{\widetilde{\tau}} \alpha(n)\right],
$$

or $\widetilde{\tau}=\tau+\varepsilon \geq \frac{\alpha(n)}{\left[\alpha\left(\ln \left|a_{n}\right|^{-1 / n}\right)\right]^{\rho}}(1+o(1))$.

Now proceeding to limits, we can get

$$
\tau \geq \limsup _{n \rightarrow \infty} \frac{\alpha(n)}{\left[\alpha\left(\ln \left|a_{n}\right|^{-1 / n}\right)\right]^{\rho}},
$$

the above inequality obviously holds when $\tau=\infty$.

Case II: Conversely, let

$$
\limsup _{n \rightarrow \infty} \frac{\alpha(n)}{\left[\alpha\left(\ln \left|a_{n}\right|^{-1 / n}\right)\right]^{\rho}}=B .
$$

We suppose $B<\infty$, then for every $\varepsilon>0$ and for all $n \geq n_{0}$, we have $\alpha(n)<(B+\varepsilon)\left[\alpha\left(\ln \left|a_{n}\right|^{-1 / n}\right)\right]^{\rho}=\widetilde{B}\left[\alpha\left(\ln \left|a_{n}\right|^{-1 / n}\right)\right]^{\rho}$, where $\widetilde{B}=B+\varepsilon$, hence $\alpha^{-1}\left(\left[\frac{1}{\widehat{B}} \alpha(n)\right]^{\frac{1}{\rho}}\right)<-\frac{1}{n} \ln \left|a_{n}\right|$. That is to say $\forall \varepsilon>0, \exists n_{0}>0$, when $n>n_{0}$, we have

$$
\left|a_{n}\right|<e^{-n \alpha^{-1}\left\{\left[\frac{1}{\widetilde{B}} \alpha(n)\right]^{\frac{1}{\rho}}\right\}} .
$$

In addition, when $r$ is large enough, there exists $S\left(S \in N^{+}\right)>n_{0}, k>0$, so that

$$
S \leq \alpha^{-1}\left\{\left[\widetilde{B}[\alpha(\ln r+k)]^{\rho}\right\} \leq S+1\right.
$$

we have

$$
\begin{gathered}
M(r) \leq \sum_{n=0}^{n_{0}}\left|a_{n}\right| r^{n}+\sum_{n=n_{0}+1}^{S}\left|a_{n}\right| r^{n}+\sum_{n=S+1}^{\infty}\left|a_{n}\right| r^{n}=A_{1}+A_{2}+A_{3} . \\
A_{2} \leq \sum_{n=n_{0}+1}^{S} e^{-n \alpha^{-1}\left\{\left[\frac{1}{B} \alpha(n)\right]^{\frac{1}{\rho}}\right\}} \cdot r^{\alpha^{-1}\left\{\left[\widetilde{B}[\alpha(\ln r+k)]^{\rho}\right\}\right.} \leq C r^{\alpha^{-1}\left\{\left[\widetilde{B}[\alpha(\ln r+k)]^{\rho}\right\}\right.},
\end{gathered}
$$


but in $A_{3}^{\prime}$, we can see that $n>\alpha^{-1}\left\{\left[\widetilde{B}[\alpha(\ln r+k)]^{\rho}\right\}\right.$, then

$$
\ln r<\alpha^{-1}\left\{\left[\frac{1}{\widetilde{B}} \alpha(n)\right]^{1 / \rho}\right\}-k,
$$

from (8) and the above inequality, it following that

$$
\begin{aligned}
& A_{3} \leq \sum_{n=S+1}^{\infty} e^{-n \alpha^{-1}\left\{\left[\frac{1}{\tilde{B}} \alpha(n)\right]^{\frac{1}{\rho}}\right\}} \cdot e^{n \ln r} \\
\leq & \sum_{n=S+1}^{\infty} e^{-n \alpha^{-1}\left\{\left[\frac{1}{\tilde{B}} \alpha(n)\right]^{\frac{1}{\rho}}\right\}} \cdot e^{n \alpha^{-1}\left\{\left[\frac{1}{\tilde{B}} \alpha(n)\right]^{\frac{1}{\rho}}\right\}-n k}=\sum_{n=S+1}^{\infty} e^{-n k}<C .
\end{aligned}
$$

Therefore $M(r)<(1+o(1)) C r^{\alpha^{-1}\left\{\left[\widetilde{B}[\alpha(\ln r+k)]^{\rho}\right\}\right.}$, then

$$
\alpha(\ln M(r)) \leq(1+o(1)) \alpha\left\{\ln r \alpha^{-1}\left\{\widetilde{B}[\alpha(\ln r+k)]^{\rho}\right\}\right\} .
$$

In view of Lemma 4 ,

$$
\limsup _{r \rightarrow \infty} \frac{\alpha(\ln M(r))}{[\alpha(\ln r)]^{\rho}} \leq B
$$

So from Case I and Case II, the proof is completed.

\section{The proof of theorem 3}

Case I: When $p=1, \mathrm{R}$. Ganti and G. S. Srivastava ${ }^{[5]}$ has been proved that

$$
\tau=\limsup _{n \rightarrow \infty} \frac{\alpha\left(\frac{n}{\rho}\right)}{\left[\alpha\left(\frac{\rho}{\rho-1} \ln \left|E_{n}(B(p, q, k) ; f)\right|^{-1 / n}\right)\right]^{\rho-1}} .
$$

It follows (4) that,

$$
\begin{aligned}
& \limsup _{n \rightarrow \infty} \frac{\alpha\left(\frac{n}{\rho}\right)}{\left[\alpha\left(\frac{\rho}{\rho-1} \ln \left|E_{n}(B(p, q, k) ; f)\right|^{-1 / n}\right)\right]^{\rho-1}}=\limsup _{n \rightarrow \infty} \frac{\alpha\left(\frac{n}{\rho}\right)}{\alpha(n)} \\
& \cdot \frac{\left[\alpha\left(\ln \left|E_{n}(B(p, q, k) ; f)\right|^{-1 / n}\right)\right]^{\rho-1}}{\left[\alpha\left(\frac{\rho}{\rho-1} \ln \left|E_{n}(B(p, q, k) ; f)\right|^{-1 / n}\right)\right]^{\rho-1}} \cdot \frac{\alpha(n)}{\left[\alpha\left(\ln \left|E_{n}(B(p, q, k) ; f)\right|^{-1 / n}\right)\right]^{\rho-1}} \\
& =\limsup _{n \rightarrow \infty} \frac{\alpha(n)}{\left[\alpha\left(\ln \left|E_{n}(B(p, q, k) ; f)\right|^{-1 / n}\right)\right]^{\rho-1}} .
\end{aligned}
$$

Case II: When $p=2,3 \cdots$, we prove this part in two steps. Fristly, we consider the space $B(p, q, k), q=2,0<p<2$, and $k \geq 1$. Let $f(z) \in B(p, q, k)$ be of generalized type $\tau$ with generalized order $\rho$. Then from theorem 2, we have

$$
\limsup _{n \rightarrow \infty} \frac{\alpha(n)}{\left.\left[\left.\alpha\left(\ln \mid a_{n}\right)\right|^{-1 / n}\right)\right]^{\rho}}=\tau .
$$


For a given $\varepsilon>0$, and all $n>m=m(\varepsilon)$, we have

$$
\left|a_{n}\right| \leq e^{-n \alpha^{-1}\left\{\left[\frac{1}{\tilde{\tau}} \alpha(n)\right]^{1 / \rho}\right\}},
$$

where $\widetilde{\tau}=\tau+\varepsilon$.

Let $g_{n}(f, z)=\sum_{j=0}^{n} a_{j} z^{j}$ be the $n^{t h}$ partial sum of the Taylor series of the function $f(z)$. Following ${ }^{[6]}$, we can get

$$
E_{n}(B(p, 2, k) ; f) \leq B^{1 / k}[(n+1) k+1 ; k(1 / p-1 / 2)]\left\{\sum_{j=n+1}^{\infty}\left|a_{j}\right|^{2}\right\}^{1 / 2},
$$

where $B(a, b)(a, b>0)$ denotes the bata function. By using (10), we get

$$
E_{n}(B(p, 2, k) ; f) \leq \frac{B^{1 / k}[(n+1) k+1 ; k(1 / p-1 / 2)]}{\exp \left\{(n+1) \alpha^{-1}\left\{\left[\frac{1}{\tilde{\tau}} \alpha(n+1)\right]^{1 / \rho}\right\}\right\}}\left\{\sum_{j=n+1}^{\infty} \psi_{j}^{2}(\alpha)\right\}^{1 / 2}
$$

where $\psi_{j}(\alpha) \cong \frac{\exp \left\{(n+1) \alpha^{-1}\left\{\left[\frac{1}{\tilde{\tau}} \alpha(n+1)\right]^{1 / \rho}\right\}\right\}}{\exp \left\{j \alpha^{-1}\left\{\left[\frac{1}{\tilde{\tau}} \alpha(j)\right]^{1 / \rho}\right\}\right\}}$, set $\psi(\alpha) \cong e^{-\alpha^{-1}\left\{\left[\frac{1}{\tilde{\tau}} \alpha(1)\right]^{1 / \rho}\right\}}$, since $\alpha(x)$ is increasing and $j>n+1$, we obtain

$$
\psi_{j}(\alpha) \leq e^{\{(n+1)-j\} \alpha^{-1}\left\{\left[\frac{1}{\tilde{\tau}} \alpha(n+1)\right]^{1 / \rho}\right\}}<\psi^{j-(n+1)}(\alpha) .
$$

Owing to $\psi(\alpha)<1$, from (11) and (12), we have

$$
E_{n}(B(p, 2, k) ; f) \leq \frac{B^{1 / k}[(n+1) k+1 ; k(1 / p-1 / 2)]}{\left(1-\psi^{2}(\alpha)\right)^{1 / 2}\left\{\exp \left\{(n+1) \alpha^{-1}\left\{\left[\frac{1}{\tau} \alpha(n+1)\right]^{1 / \rho}\right\}\right\}\right\}}
$$

For $n>m,(13)$ yields

$$
\widetilde{\tau}=\tau+\varepsilon \geq \frac{\alpha(n+1)}{\left\{\alpha\left(\frac{n}{n+1}\left\{\ln \left|E_{n}\right|^{-1 / n}+\ln \left(\frac{B^{1 / k}[(n+1) k+1 ; k(1 / p-1 / 2)]}{\left(1-\psi^{2}(\alpha)\right)^{1 / 2}}\right)^{1 / n}\right\}\right)\right\}^{\rho}} .
$$

Now

$$
B[(n+1) k+1 ; k(1 / p-1 / 2)]=\frac{\Gamma((n+1) k+1) \Gamma(k(1 / p-1 / 2))}{\Gamma((n+1 / 2+1 / p) k+1)},
$$

therefore

$$
B[(n+1) k+1 ; k(1 / p-1 / 2)]
$$




$$
\simeq \frac{e^{-[(n+1) k+1]}[(n+1) k+1]^{(n+1) k+3 / 2} \Gamma(1 / p-1 / 2)}{e^{[(n+1 / 2+1 / p) k+1]}[(n+1 / 2+1 / p) k+1]^{(n+1 / 2+1 / p) k+3 / 2}},
$$

thus

$$
\{B[(n+1) k+1 ; k(1 / p-1 / 2)]\}^{1 /(n+1)} \cong 1 .
$$

Now proceeding to limits, we have

$$
\tau \geq \limsup _{n \rightarrow \infty} \frac{\alpha(n)}{\left[\alpha\left(\ln \left|E_{n}\right|^{-1 / n}\right)\right]^{\rho}} .
$$

For reverse inequality, by ${ }^{[6]}$, we have,

$$
\left|a_{n+1}\right| B^{1 / k}[(n+1) k+1 ; k(1 / p-1 / 2)] \leq E_{n}(B(p, 2, k) ; f) .
$$

Then for sufficiently large $n$, we can get

$$
\begin{aligned}
& \frac{\alpha(n)}{\left[\alpha\left(\ln \left|E_{n}\right|^{-1 / n}\right)\right]^{\rho}} \\
\geq & \frac{\alpha(n)}{\left\{\alpha\left\{\ln \left|a_{n+1}\right|^{-1 / n}+\ln \left(B^{-1 / n k}[(n+1) k+1 ; k(1 / p-1 / 2)]\right)\right\}\right\}^{\rho}} \\
\geq & \frac{\alpha(n)}{\left\{\alpha\left\{\ln \left|a_{n}\right|^{-1 / n}+\ln \left(B^{-1 / n k}[(n+1) k+1 ; k(1 / p-1 / 2)]\right)\right\}\right\}^{\rho}} .
\end{aligned}
$$

by applying limits and in view of (9), we obtain

$$
\limsup _{n \rightarrow \infty} \frac{\alpha(n)}{\left[\alpha\left(\ln \left|E_{n}\right|^{-1 / n}\right)\right]^{\rho}} \geq \tau .
$$

It follow from (15) and (17) that,

$$
\limsup _{n \rightarrow \infty} \frac{\alpha(n)}{\left[\alpha\left(\ln \left|E_{n}\right|^{-1 / n}\right)\right]^{\rho}}=\tau .
$$

In the second steps, we consider the space $B(p, q, k)$ for $0<p<q, q \neq 2$, and $q, k \geq 1$. Gvaradze ${ }^{[7]}$ show that, for $p \geq p_{1}, q \leq q_{1}$, and $k \leq k_{1}$, if at least one of the inequality is strict, then the strict inclusion $B(p, q, k) \subset B\left(p_{1}, q_{1}, k_{1}\right)$ holds and the following relation is true:

$$
\|f\|_{p_{1}, q_{1}, k_{1}} \leq 2^{1 / q-1 / q_{1}}[k(1 / p-1 / q)]^{1 / k-1 / k_{1}}\|f\|_{p, q, k} .
$$

For any function $f(z) \in B(p, q, k)$, the last relation yields

$$
E_{n}\left(B\left(p_{1}, q_{1}, k_{1}\right) ; f\right) \leq 2^{1 / q-1 / q_{1}}[k(1 / p-1 / q)]^{1 / k-1 / k_{1}} E_{n}(B(p, q, k) ; f) .
$$


For the general case $B(p, q, k), q \neq 2$, we prove the necessity of condition (10). Let $f(z) \in B(p, q, k)$ be an entire transcendental function having finite generalized order $\rho(\alpha ; f)$ whose generalized type is defined by (9). Using the relation (10), for $n>m$ we estimate the value of the best polynomial approximation as follows

$$
E_{n}(B(p, q, k) ; f)=\left\|f-g_{n}(f)\right\|_{p, q, k} \leq\left\{\int_{0}^{1}(1-r)^{k(1 / p-1 / q)-1} M_{q}^{k} d r\right\}^{1 / k}
$$

Let

$$
|f|^{q}=\left|\sum a_{n} z^{n}\right|^{q} \leq\left(\sum\left|a_{n} r^{n}\right|\right)^{q} \leq\left(r^{n+1} \sum_{k=n+1}^{\infty}\left|a_{k}\right|\right)^{q}
$$

hence

$$
\begin{aligned}
E_{n}(B(p, q, k) ; f) & \leq B^{1 / k}[(n+1) k+1 ; k(1 / p-1 / q)] \sum_{k=n+1}^{\infty}\left|a_{k}\right| \\
& \leq \frac{B^{1 / k}[(n+1) k+1 ; k(1 / p-1 / q)]}{(1-\psi(\alpha))\left[\exp \left\{(n+1) \alpha^{-1}\left\{\left[\frac{1}{\tau} \alpha(n+1)\right]^{1 / \rho}\right\}\right\}\right]} .
\end{aligned}
$$

For $n>m$, from the above inequality, we have

$$
\widetilde{\tau}=\tau+\varepsilon \geq \frac{\alpha(n+1)}{\left\{\alpha\left(\frac{n}{n+1}\left\{\ln \left|E_{n}\right|^{-1 / n}+\ln \left(\frac{B^{1 / k}[(n+1) k+1 ; k(1 / p-1 / 2)]}{(1-\psi(\alpha))}\right)^{1 / n}\right\}\right)\right\}^{\rho}} .
$$

Since $\psi(\alpha)<1$, and $\alpha \in \Lambda$, proceeding to limits and using (14), we have

$$
\tau \geq \limsup _{n \rightarrow \infty} \frac{\alpha(n)}{\left[\alpha\left(\ln \left|E_{n}\right|^{-1 / n}\right)\right]^{\rho}} .
$$

For the reverse inequality, let $0<p<q<2$ and $k, q \geq 1$. By (19), where $p_{1}=P, q_{1}=2$, and $k_{1}=k$, and the condition (10) is already proved for the space $B(p, 2, k)$, we obtain

$$
\limsup _{n \rightarrow \infty} \frac{\alpha(n)}{\left[\alpha\left(\ln \left|E_{n}(B(p, q, k) ; f)\right|^{-1 / n}\right)\right]^{\rho}} \geq \limsup _{n \rightarrow \infty} \frac{\alpha(n)}{\left[\alpha\left(\ln \left|E_{n}(B(p, 2, k) ; f)\right|^{-1 / n}\right)\right]^{\rho}}=\tau \text {. }
$$

Now we let $0<p \leq 2<q$, then $M_{2}(r, f) \leq M_{q}(r, f), \quad 0<r<1$, therefore

$$
E_{n}(B(p, q, k) ; f) \geq\left|a_{n+1}\right| B^{1 / k}[(n+1) k+1 ; k(1 / p-1 / q)],
$$

then for $n$ is large enough, we have

$$
\frac{\alpha(n)}{\left[\alpha\left(\ln \left|E_{n}\right|^{-1 / n}\right)\right]^{\rho}}
$$




$$
\begin{aligned}
& \geq \frac{\alpha(n)}{\left\{\alpha\left\{\ln \left|a_{n+1}\right|^{-1 / n}+\ln \left(B^{-1 / n k}[(n+1) k+1 ; k(1 / p-1 / q)]\right)\right\}\right\}^{\rho}} \\
& \geq \frac{\alpha(n)}{\left\{\alpha\left\{\ln \left|a_{n}\right|^{-1 / n}+\ln \left(B^{-1 / n k}[(n+1) k+1 ; k(1 / p-1 / q)]\right)\right\}\right\}^{\rho}} .
\end{aligned}
$$

By applying limits and from (9), we obtain

$$
\limsup _{n \rightarrow \infty} \frac{\alpha(n)}{\left[\alpha\left(\ln \left|E_{n}\right|^{-1 / n}\right)\right]^{\rho}} \geq \limsup _{n \rightarrow \infty} \frac{\alpha(n)}{\left[\alpha\left(\ln \left|a_{n}\right|^{-1 / n}\right)\right]^{\rho}}=\tau .
$$

Now we assume that $2 \leq p<q$. Set $q_{1}=q, k_{1}=k$, and $0<p_{1}<2$ in the inequality (19), where $p_{1}$ is an arbitrary fixed number. Substituting $p_{1}$ for $p$ in (20), we can get

$$
E_{n}(B(p, q, k) ; f) \geq\left|a_{n+1}\right| B^{1 / k}\left[(n+1) k+1 ; k\left(1 / p_{1}-1 / q\right)\right] .
$$

From the above inequality and applying the same analogy as in the previous case $0<p \leq 2<q$, for sufficiently large $n$, we obtain

$$
\geq \frac{\frac{\alpha(n)}{\left[\alpha\left(\ln \left|E_{n}\right|^{-1 / n}\right)\right]^{\rho}}}{\left\{\alpha\left\{\ln \left|a_{n}\right|^{-1 / n}+\ln \left(B^{-1 / n k}\left[(n+1) k+1 ; k\left(1 / p_{1}-1 / q\right)\right]\right)\right\}\right\}^{\rho}},
$$

by applying limits and in view of (9), we obtain

$$
\limsup _{n \rightarrow \infty} \frac{\alpha(n)}{\left[\alpha\left(\ln \left|E_{n}\right|^{-1 / n}\right)\right]^{\rho}} \geq \tau .
$$

From the relations $(15),(17)$ and the above inequality, we obtain the required relation (18). So from Case I and Case II, the proof is completed.

\section{The proof of theorem 4}

Case I: When $p=1$, R. Ganti and G. S. Srivastava ${ }^{[5]}$ has been proved that

$$
\limsup _{n \rightarrow \infty} \frac{\alpha\left(\frac{n}{\rho}\right)}{\left[\alpha\left(\frac{\rho}{\rho-1} \ln \left|E_{n}\left(H_{q} ; f\right)\right|^{-1 / n}\right)\right]^{\rho-1}}=\xi(\alpha) .
$$

It follows (4) that,

$$
\limsup _{n \rightarrow \infty} \frac{\alpha\left(\frac{n}{\rho}\right)}{\left[\alpha\left(\frac{\rho}{\rho-1} \ln \left|E_{n}\left(H_{q} ; f\right)\right|^{-1 / n}\right)\right]^{\rho-1}}
$$




$$
\begin{aligned}
=\quad & \limsup _{n \rightarrow \infty} \frac{\alpha\left(\frac{n}{\rho}\right)}{\alpha(n)} \cdot \frac{\left[\alpha\left(\ln \left|E_{n}\left(H_{q} ; f\right)\right|^{-1 / n}\right)\right]^{\rho-1}}{\left[\alpha\left(\frac{\rho}{\rho-1} \ln \left|E_{n}\left(H_{q} ; f\right)\right|^{-1 / n}\right)\right]^{\rho-1}} \\
& \cdot \frac{\alpha(n)}{\left[\alpha\left(\ln \left|E_{n}\left(H_{q} ; f\right)\right|^{-1 / n}\right)\right]^{\rho-1}} \\
=\quad & \limsup _{n \rightarrow \infty} \frac{\alpha(n)}{\left[\alpha\left(\ln \left|E_{n}\left(H_{q} ; f\right)\right|^{-1 / n}\right)\right]^{\rho-1}} .
\end{aligned}
$$

Case II: When $p=2,3 \cdots$, Let $f(z)=\sum_{n=0}^{\infty} a_{n} z^{n}$ be an entire transcendental function having finite generalized order $\rho$ and generalized type $\tau$. Since $\limsup _{n \rightarrow \infty} \sqrt[n]{\left|a_{n}\right|}=0, f(z) \in B(p, q, k)$, where $0<P<q \leq \infty$ and $q, k \geq 1$. From relation (5), we have

$$
E_{n}(B(q / 2, q, q) ; f) \leq \varsigma_{q} E_{n}\left(H_{q} ; f\right)(1 \leq q<\infty)
$$

where $\varsigma_{q}$ is an constant independent of $n$ and $f$. In the case of Hardy space $H_{\infty}$

$$
E_{n}(B(p, \infty, \infty) ; f) \leq E_{n}\left(H_{q} ; f\right)(1<q<\infty)
$$

since

$$
\begin{aligned}
\xi(\alpha, f) & =\limsup _{n \rightarrow \infty} \frac{\alpha(n)}{\left[\alpha\left(\ln \left|E_{n}\left(H_{q} ; f\right)\right|^{-1 / n}\right)\right]^{\rho}} \\
& \geq \limsup _{n \rightarrow \infty} \frac{\alpha(n)}{\left[\alpha\left(\ln \left|E_{n}(B(q / 2, q, q) ; f)\right|^{-1 / n}\right)\right]^{\rho}} \\
& \geq \tau(1 \leq q<\infty) .
\end{aligned}
$$

Using estimate (23) we prove the above inequality in the case $q=\infty$.

Conversely, Let the generalized type $\tau$ of an an entire transcendental function $f(z)$ having finite generalized order $\rho$ as follows and using the relation (10), we have

$$
\begin{aligned}
& E_{n}\left(H_{q} ; f\right)=\left\|f-g_{n}(f)\right\|_{H_{q}} \leq \sum_{j=n+1}^{\infty}\left|a_{j}\right| \\
& \leq \frac{1}{\exp \left\{(n+1) \alpha^{-1}\left\{\left[\frac{1}{\tilde{\tau}} \alpha(n+1)\right]^{1 / \rho}\right\}\right\}}\left\{\sum_{j=n+1}^{\infty} \psi_{j}(\alpha)\right\} .
\end{aligned}
$$

On account of (12)

$$
E_{n}\left(H_{q} ; f\right)=\left\|f-g_{n}(f)\right\|_{H_{q}}
$$




$$
\leq \frac{1}{(1-\psi(\alpha))\left[\exp \left\{(n+1) \alpha^{-1}\left\{\left[\frac{1}{\tilde{\tau}} \alpha(n+1)\right]^{1 / \rho}\right\}\right\}\right]}
$$

this is to say

$$
\widetilde{\tau}=\tau+\varepsilon \geq \frac{\alpha(n+1)}{\left\{\alpha\left(\ln \left|E_{n}\left(H_{q} ; f\right)\right|^{-1 / n+1}+\ln \left[(1-\psi(\alpha))^{-1 / n+1}\right]\right)\right\}^{\rho}} .
$$

Now proceeding to limits, since $\psi(\alpha)<1$ and by applying the relation (4), we have

$$
\xi(\alpha, f) \leq \limsup _{n \rightarrow \infty} \frac{\alpha(n)}{\left[\alpha\left(\ln \left|E_{n}\left(H_{q} ; f\right)\right|^{-1 / n}\right)\right]^{\rho}},
$$

so $\xi(\alpha, f)=\tau$, this prove is completed.

Remark: An analogy of Theorem 4 for Bergman Spaces follows from (5) and from Theorem 3 for $q=\infty$.

\section{Acknowledgments}

The authors would like to thank the anonymous reviewers and editor for their valuable comments and suggestions to improve the quality of the paper. This project is supported by the National Natural Science Foundation of China (11661044).

\section{References}

[1] S.M. Shah, Polynomial approximation of an entire function and generalized orders, Journal of approximation theory, 4 (1977) 315-324, doi: 10.1016/0021-9045(77)90095-8.

[2] S.K. Bajpai, G.P. Kapoor, and O.P. Juneja, On entire function of fast growth, Transactions of the american mathematical society, 203 (1975) 275-297, doi: 10.1090/S00029947-1975-0372200-0.

[3] G.P. Kapoor, A. Nautiyal, Polynomial approximation of an entire function of slow growth, Journal of approximation theory, 32 (1981) 64-75, doi: 10.1016/0021-9045(81)90022-8.

[4] A. Nautiyal, G.P. Kapoor, On the generalized orders of an entire function of slow growth, Indian journal of pure and applied mathematics, 11 (1982) 1246-1252.

[5] R. Ganti, G.S. Srivastava, Approxiamtion of entire function of slow growth, General mathematics, 14 (2006) 59-76.

[6] S.B. Vakarchuk, S.I. Zhir, On some problem of polynomial approximation of entire transcendental function, Ukrainian mathematical journal, 9 (2002) 1393-1401, doi: 10.1023/A:1023407416027.

[7] M.I. Gvaradze, On the class of spaces of analytic functions. Matematicheskie zametki, 2 (1977), 141-150. doi: 10.1007/BF02320544. 
[8] S.B. Vakarchuk, On the best polynomial approximation in some Banach spaces for functions analytical in the unit disc, Mathematical notes, 4 (1994) 338-343, doi: 10.1007/BF02112471.

[9] Y.Y. Kong , H.L. Gan, On orders and types of Dirichlet series of slow growth, Turkish journal of mathematics, 1 (2010) 1-11, doi: 10.3906/mat-0810-17. 
